OPEN ACCESS

Edited by:

Thea Magrone,

University of Bari Aldo Moro, Italy

Reviewed by:

Nobuhiko Kamada,

University of Michigan Health System,

United States

Alberto Finamore

Council for Agricultural and

Economics Research, Italy

${ }^{*}$ Correspondence:

Jun Kunisawa

kunisawa@nibiohn.go.jp

Specialty section:

This article was submitted to

Nutritional Immunology,

a section of the journal

Frontiers in Nutrition

Received: 04 February 2019

Accepted: 14 March 2019

Published: 10 April 2019

Citation:

Saika A, Nagatake T and Kunisawa J (2019) Host- and Microbe-Dependent

Dietary Lipid Metabolism in the

Control of Allergy, Inflammation, and

Immunity. Front. Nutr. 6:36.

doi: 10.3389/fnut.2019.00036

\section{Host- and Microbe-Dependent Dietary Lipid Metabolism in the Control of Allergy, Inflammation, and Immunity}

\author{
Azusa Saika ${ }^{1,2}$, Takahiro Nagatake ${ }^{1}$ and Jun Kunisawa ${ }^{1,2,3,4,5 *}$ \\ ${ }^{1}$ Laboratory of Vaccine Materials, Center for Vaccine and Adjuvant Research, and Laboratory of Gut Environmental System, \\ National Institutes of Biomedical Innovation, Health and Nutrition, Osaka, Japan, ${ }^{2}$ Graduate School of \\ Pharmaceutical Sciences, Osaka University, Osaka, Japan, ${ }^{3}$ International Research and Development Center for Mucosal \\ Vaccines, Institute of Medical Science, University of Tokyo, Tokyo, Japan, ${ }^{4}$ Graduate School of Medicine, Graduate School of \\ Dentistry, Osaka University, Osaka, Japan, ${ }^{5}$ Graduate School of Medicine, Kobe University, Kobe, Japan
}

The intestine is the largest immune organ in the body, provides the first line of defense against pathogens, and prevents excessive immune reactions to harmless or beneficial non-self-materials, such as food and intestinal bacteria. Allergic and inflammatory diseases in the intestine occur as a result of dysregulation of immunological homeostasis mediated by intestinal immunity. Several lines of evidence suggest that gut environmental factors, including nutrition and intestinal bacteria, play important roles in controlling host immune responses and maintaining homeostasis. Among nutritional factors, $\omega 3$ and $\omega 6$ essential polyunsaturated fatty acids (PUFAs) profoundly influence the host immune system. Recent advances in lipidomics technology have led to the identification of lipid mediators derived from $\omega 3-$ and $\omega 6$-PUFAs. In particular, lipid metabolites from $\omega 3$-PUFAs (e.g., eicosapentaenoic acid and docosahexaenoic acid) have recently been shown to exert anti-allergic and anti-inflammatory responses; these metabolites include resolvins, protectins, and maresins. Furthermore, a new class of anti-allergic and anti-inflammatory lipid metabolites of 17,18-epoxyeicosatetraenoic acid has recently been identified in the control of allergic and inflammatory diseases in the gut and skin. Although these lipid metabolites were found to be endogenously generated in the host, accumulating evidence indicates that intestinal bacteria also participate in lipid metabolism and thus generate bioactive unique lipid mediators. In this review, we discuss the production machinery of lipid metabolites in the host and intestinal bacteria and the roles of these metabolites in the regulation of host immunity.

Keywords: lipid metabolites, dietary oil, intestinal immunity, inflammation, allergy, intestinal bacteria

\section{INTRODUCTION}

Lipid composition in organisms differs among species, in accordance with the expression levels of metabolic enzymes and dietary habits. Marine phytoplankton and seaweeds produce a large amount of the $\omega 3$-polyunsaturated fatty acids (PUFAs) eicosapentaenoic acid (EPA) and docosahexaenoic acid (DHA) (1). Although fish do not generate EPA and DHA per se, they accumulate EPA and DHA by eating phytoplankton (1). In plants, linseed and perilla contain large amounts of 
$\alpha$-linolenic acid, a precursor of EPA and DHA. In contrast, soybean oil and sesame oil contain copious quantities of the $\omega 6$ PUFA linoleic acid. The difference in the fatty acid composition of plants depends on the expression levels and activities of metabolic enzymes such as $\Delta 12$-desaturase and $\Delta 15$-desaturase, which are involved in the generation of linoleic acid and $\alpha$ linolenic acid, respectively $(2,3)$. Because mammals do not have either $\Delta 12$ or $\Delta 15$-desaturase, $\omega 3$ - and $\omega 6$-PUFAs are categorized as essential fatty acids that must be obtained from the diet (3). Therefore, the balance of $\omega 3$ and $\omega 6$ lipids in the body largely depends on the quality of the dietary lipid consumed.

The beneficial effect of dietary $\omega 3$-PUFAs on human health was first reported in an epidemiological study in 1978 in which Greenland Eskimos, who consume high $\omega 3$-PUFA diets that include fish, were found to have a lower mortality from coronary heart disease than Danes and Americans, who eat much less w3PUFAs (4). Since then, accumulating evidence indicates that EPA and DHA have beneficial effects on the inhibition of various types of inflammatory and allergic diseases, including cardiovascular disease, Alzheimer's disease, rheumatoid arthritis, inflammatory bowel disease, atopic dermatitis, asthma, and food allergy (5-13). Recent developments in analytical technology, including liquid chromatography (LC) and mass spectrometry (MS), have enabled us to identify EPA- and DHA-derived pro-resolving lipid mediators (SPMs), including resolvins (Rvs), protectins (PDs), maresins (MaRs), and 17,18-epoxyeicosatetraenoic acid $(17,18$-EpETE) for inhibition of inflammatory and allergic diseases $(7,14)$.

Dietary lipids are metabolized not only by mammalian enzymes but also by bacterial enzymes. Microorganisms can generate unique lipid metabolites such as conjugated linoleic acids, hydroxy fatty acids, and oxo fatty acids. These bacteriaproduced lipid metabolites show biological activity in the context of host health and diseases $(15,16)$. Here, we review our current understanding of $\omega 3$ - and $\omega 6$-PUFA-derived lipid mediators in the control of inflammatory and allergic diseases.

\section{$\omega 6$ FATTY ACID METABOLITES HAVE OPPOSING ROLES IN PRO-AND ANTI-INFLAMMATION}

Dietary lipids are metabolized in the body to lipid mediators, which regulate host immune systems. Arachidonic acid (AA) is

Abbreviations: 12-HHT, 12-hydroxy-heptadecatrienoic acid; 14,15-EpETE, 14,15-epoxyeicosatetraenoic acid; 17,18-EpETE, 17,18-epoxyeicosatetraenoic acid; 17,18-diHETE, 17,18-dihydroxy-eicosatetraenoic acid; AA, arachidonic acid; CHS, contact hypersensitivity; CLA, conjugated linoleic acid; COX, cyclooxygenase; CRTH2, chemoattractant receptor-homologous molecule expressed on Th2 cells; CYP, cytochrome P450; DC, dendritic cell; DHA, docosahexaenoic acid; DSS, dextran sodium sulfate; EPA, eicosapentaenoic acid; GPR, G-protein-coupled receptor; HYA, 10-hydroxy-cis-12-octadecenoic acid; HYB, 10-hydroxy-octadecanoic acid; HYC, 10-hydroxy-trans-11-octadecenoic acid; IL, interleukin; KetoA, 10-oxo-cis-12-octadecenoic acid; KetoB, 10oxo-octadecanoic acid; KetoC, 10-oxo-trans-11-octadecenoic acid; LC, liquid chromatography; LOX, lipoxygenase; LT, leukotriene; MaR, maresin; MCRA, myosin cross-reactive antigen; MS, mass spectrometry; NF, nuclear factor; OVA, ovalbumin; PD, protectin; PG, prostaglandin; PPAR, peroxisome proliferatoractivated receptor; PUFA, polyunsaturated fatty acid; Rv, resolvin; SPM, specialized pro-resolving lipid mediator; TNF, tumor necrosis factor; TX, thromboxane. a metabolite of linoleic acid, and functions as a direct precursor of bioactive lipid mediators, which are known as eicosanoids. In addition to its biosynthesis in the body from linoleic acid, AA can be obtained from dietary sources, such as meat and eggs. AA is metabolized by cyclooxygenase (COX), lipoxygenase (LOX), and cytochrome P450 (CYP), and then converted into lipid mediators, including prostaglandins (PGs), leukotrienes (LTs), thromboxanes (TXs), and lipoxins (LXs) (Figure 1) (17). These AA-derived lipid meditators have both pro- and antiinflammatory effects in the intestine.

AA is converted into $\mathrm{LTB}_{4}$ by LOX activity. The $\mathrm{LTB}_{4}{ }^{-}$ BLT1 axis plays a key role in the development of inflammatory diseases including inflammatory bowel disease by stimulating the recruitment of inflammatory cells and the production of pro-inflammatory cytokines (18-20). $\mathrm{LTB}_{4}$ also activates another receptor BLT2 which is a high affinity receptor for 12-hydroxy-heptadecatrienoic acid (12-HHT). In contrast to pro-inflammatory role of BLT1, BLT2-deficient mice show transepidermal water loss, suggesting its anti-inflammatory role in the skin (21). Indeed, BLT2-mediated pathway induced the expression of claudin-4 for enhancement of epithelial barrier (21).

AA is converted into PGs by COX activity, which generate $\mathrm{PGD}_{2}$ and $\mathrm{PGE}_{2}$ as the representative lipid mediators. The $\mathrm{PGD}_{2}$-chemoattractant receptor-homologous molecule expressed on Th2 cells (CRTH2) pathway induces dextran sodium sulfate (DSS)- and trinitrobenzene sulfonic acid (TNBS)induced colitis $(22,23)$. Eosinophil infiltration into colon is inhibited by CRTH2 antagonist treatment in TNBS-induced colitis (23). In contrast to pro-inflammatory properties, the $\mathrm{PGD}_{2}$-DP axis reduces granulocyte infiltration into the colonic mucosa in the mouse model of TNBS-induced colitis and colitis-associated colorectal cancer $(24,25)$ These opposing roles of CRTH2 and DP in chemotaxis are explained by different usage of $\mathrm{G}$ proteins. CRTH2 is coupled with $\mathrm{G} \alpha_{\mathrm{i}}$ while DP is coupled with $\mathrm{G} \alpha_{s}$, which induces decreased and increased in cAMP levels, respectively (26). Consistent with these findings when $\mathrm{PGD}_{2}$ acted on neutrophils CRTH2 pathway, it induced neutrophil migration to the intestinal lamina propria in the DSS-induced colitis model (22).

$\mathrm{PGE}_{2}$ stimulates four distinct types of receptors EP1 to EP4. The PGE 2 -EP2 axis in neutrophils and tumor-associated fibroblasts promotes colon tumorigenesis by inducing expression of inflammation- and growth-related genes, including tumor necrosis factor (TNF)- $\alpha$, interleukin (IL)-6, and Wnt5A (27). In contrast to EP2-mediated carcinogenic effects, EP3-mediated signals show anti-carcinogenic effects, which are consistent with different types of $G$ protein pathways; EP2 activates $G \alpha_{s}$, while EP3 activates $\mathrm{G} \alpha_{\mathrm{i}}(27)$.

Therefore, it is suggested that the opposing roles in proand anti-inflammation of $\omega 6$-PUFAs derived lipid mediators are determined by target cell types and receptor types.

In addition to these factors, cellular source of $\mathrm{PGD}_{2}$ affects in its activity in pro- and anti-inflammation in croton oilinduced skin inflammation model (28). In the initial phase of the dermatitis when few inflammatory cells exist in the skin, endothelial cells show highest COX-2 activity and produce $\mathrm{PGD}_{2}$, which leads to DP activation on endothelial cells, 


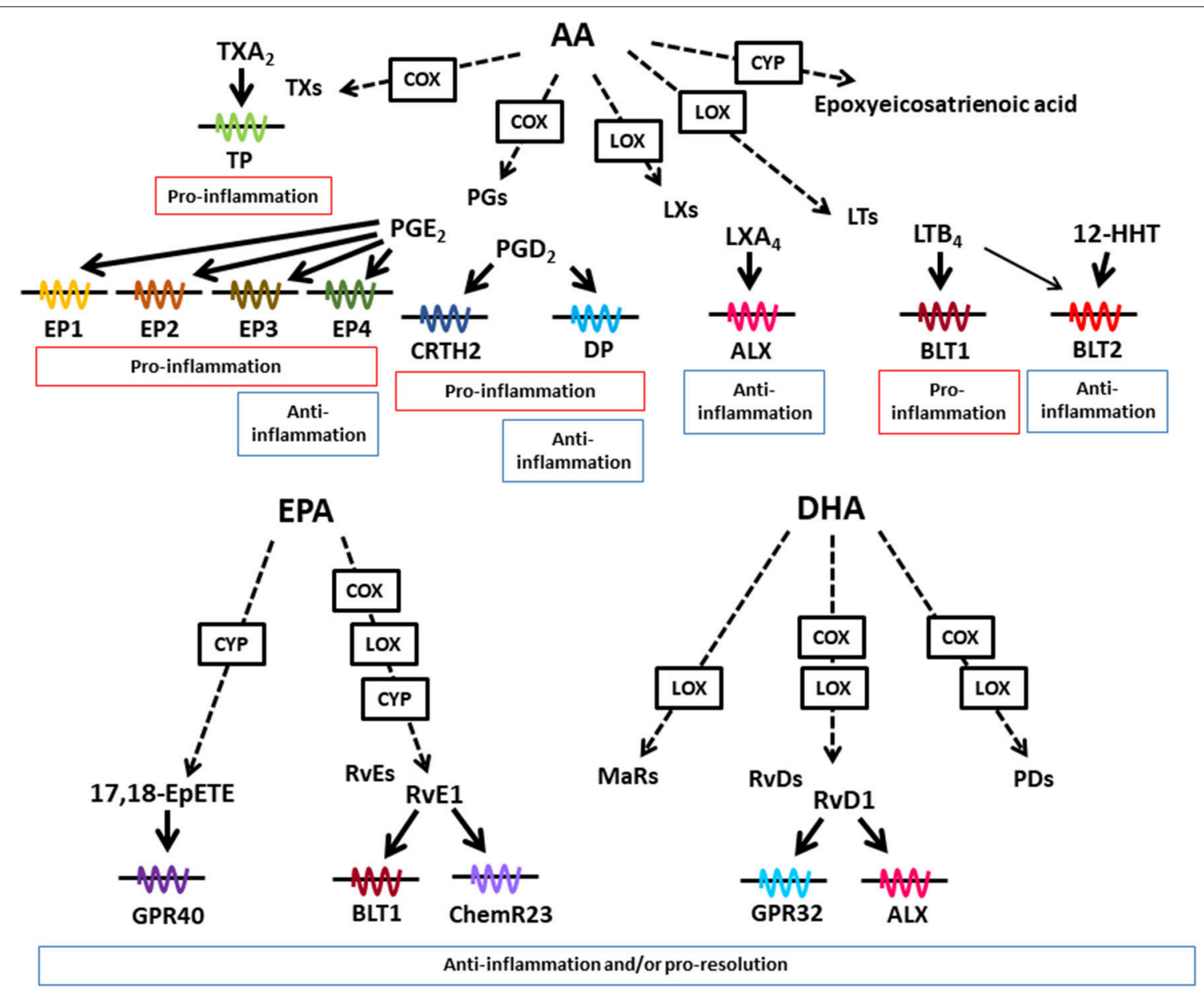

FIGURE 1 | Lipid mediators derived from AA, EPA, and DHA. Various kinds of lipid mediators are produced from $\omega 6$ - and $\omega 3$-PUFAs. AA, EPA, and DHA are converted to bioactive lipid mediators by the enzymatic activities of COX, LOX, and CYP. Lipid mediators exert their biological effects through binding to G-protein-coupled receptors. AA-derived lipid mediators have pro- and anti-inflammatory activities, whereas EPA- and DHA-derived lipid mediators exert anti-inflammatory or pro-resolution activities or both.

and inhibits vascular leakage. On the other hand, in the late phase of the dermatitis, many types of hematopoietic inflammatory cells produce $\mathrm{PGD}_{2}$, which stimulate CRTH2 on inflammatory cells for infiltration to the inflamed skin, and exacerbates skin inflammation $(28,29)$. These findings suggest that stage of inflammatory process is a determinant of the effects of AA-derived metabolites through distinct site of the mediator production.

\section{DIETARY $\omega 3$-PUFAS INHIBIT THE DEVELOPMENT OF ALLERGIC DISEASE}

We and others have shown the anti-inflammatory and antiallergic effects of dietary $\omega 3$-PUFAs $(4,7,8,12,13,30-34)$.

Fish oil is a representative $\omega 3$-PUFA-rich dietary oil which contains plenty amount of EPA and DHA. Dietary fish oil ameliorated asthma by decreasing eosinophil infiltration, mucus production, and peribronchiolar fibrosis, which was associated with inhibition of cytokine production by downregulation of nuclear factor (NF)- $\mathrm{B}$ and GATA-3 (30). These anti-allergic effects may be caused by decreased amount of $\omega 6$-PUFA-derived lipid mediators such as $\mathrm{PGD}_{2}, \mathrm{LTB}_{4}$, and $\mathrm{LTE}_{4}$ which exacerbate airway inflammation and increasing $\omega 3$-PUFA-derived lipid mediators, for example, RvD1 is reported to decrease allergic airway responses $(6,35,36)$. Further, fish oil-fed mice reduced acute allergic skin response in food allergy model sensitized by peanut and whey by reducing mucosal mast cell protease- 1 and antigen specific IgE in serum (31).

Linseed oil contains large amount of $\alpha$-linolenic acid which is converted into EPA and DHA in the body. One study reported that linseed oil-fed mice alleviated pollen-induced allergic conjunctivitis by decreasing the production of w6PUFA-derived pro-inflammatory lipid mediators, and reducing eosinophil infiltration into the conjunctiva (13). We also found that linseed oil-fed mice reduced allergic diarrhea in ovalbumin (OVA)-induced food allergy model (7). In this model, allergic diarrhea occurs as a consequence of a dominant Th2-type environment and the presence of allergen-specific serum IgE, 
which induces mast cell degranulation in the gut. We found that in linseed oil-fed mice, the Th1-Th2 balance, allergenspecific IgE level, and mast cell numbers in the gut did not change compared with those in soybean oil-fed mice in the OVA-induced food allergy model. However, we found that mast cell degranulation was profoundly inhibited in linseed oil-fed mice (7).

We also assessed fatty acid composition in intestinal tissues and found that the amounts of $\alpha$-linolenic acid and its metabolites of EPA and DHA were increased in linseed oil-fed mice when compared with those in soybean oil-fed mice (7). In contrast, linoleic acid and AA levels were higher in soybean oil-fed mice than linseed oil-fed mice (7). Imaging MS analysis revealed that increased amounts of $\alpha$-linolenic acid EPA and DHA were found in the lamina propria compartment where large numbers of immune cells such as T cells, plasma cells, and dendritic cells are present (7). These findings collectively demonstrated that the composition of essential fatty acids in dietary oils directly reflect the lipid composition in the gut, which, in turn, may influence the host immune system.

\section{(3) FATTY ACID METABOLITES HAVE ROLES IN ANTI-INFLAMMATION AND PRO-RESOLUTION}

EPA and DHA are representative $\omega 3$-PUFAs, which compete with $\mathrm{AA}$ in the AA cascade. Therefore, it has long been considered that the beneficial effects of dietary w3-PUFAs against inflammatory diseases stem from decreased amounts of AA-derived eicosanoids. In addition, recent technology developments in LC and MS have led to the identification of trace and novel lipid mediators, including Rvs, PDs, and MaRs, which are produced from EPA and DHA in the body (37). These metabolites have anti-inflammatory or pro-resolution properties (or both) and are known as SPMs (Figure 1) (37). Although the receptors for SPMs have not been fully elucidated, some SPMs have been shown to interact with specific receptors. For example, Rvs derived from EPA and DHA use distinct types of receptors. RvE1 interacts with BLT1 and ChemR23, while RvD1 interacts with G-protein-coupled receptor (GPR) 32 and $\operatorname{ALX}(38,39)$.

Examples of how SPMs affect intestinal inflammation include their involvement in the RvE1-ChemR23 axis, which actively inhibits colonic inflammation in the DSS-induced colitis model by suppressing the TNF- $\alpha$-induced nuclear translocation of NF$\kappa \mathrm{B}$ and the expression of inflammatory cytokines, including TNF- $\alpha$ and IL-12p40, from macrophages (40). Furthermore, RvE1 and PD1 enhance the resolution of inflammation by stimulating macrophage phagocytosis of apoptotic cells in zymosan-induced peritonitis $(41,42)$. MaR1 is reported to attenuate both DSS- and TNBS-induced colitis by inhibiting NF$\kappa \mathrm{B}$ activation and inflammatory cytokine production (43). Thus, multiple types of SPMs exert their anti-inflammatory properties by using different mechanisms for the regulation of colitis.

\section{7,18-EPOXYEICOSATETRAENOIC ACID IS A NEW CLASS OF ANTI-ALLERGY LIPID MEDIATOR}

As mentioned above, dietary linseed oil inhibited the development of food allergy with increased amounts of $\alpha$ linolenic acid, EPA and DHA in the intestine (7), which

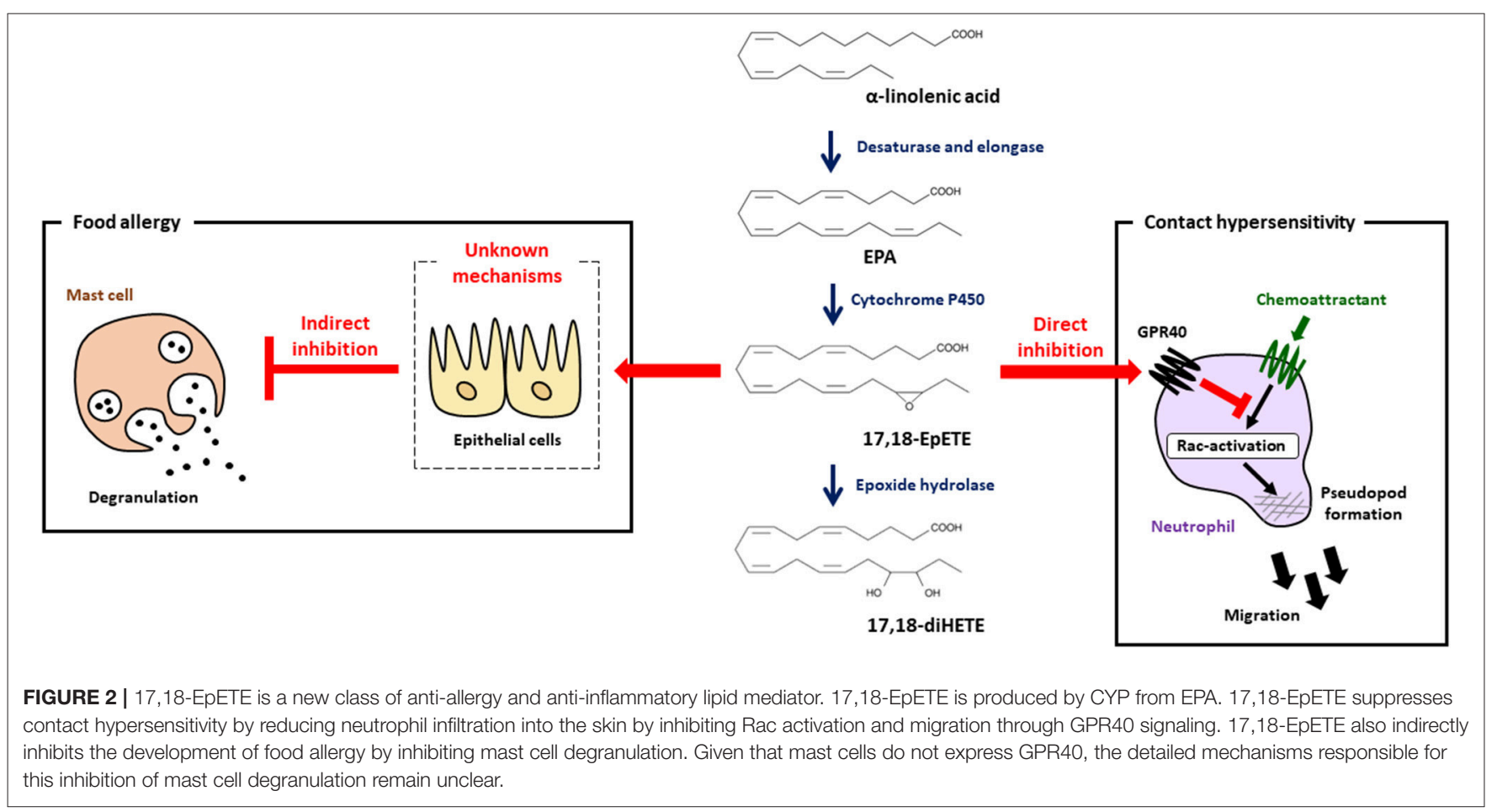


prompted us to investigate mediator profiles by using LCMS/MS analysis. We found that 17,18-EpETE was the metabolite whose levels increased the most in the gut of linseed oil-fed mice (7). When 17,18-EpETE was intraperitoneally injected into soybean oil-fed mice, development of allergic diarrhea and degranulation of mast cells were inhibited, which was similar to observation in linseed oil-fed mice (Figure 2) (7). Consistent with its action at the late stage of the allergic response, 17,18-EpETE was effective as a prophylactic and a therapeutic treatment for food allergy (7).

\section{7,18-EPETE AMELIORATES CONTACT HYPERSENSITIVITY THROUGH GPR40-MEDIATED INHIBITION OF NEUTROPHIL MIGRATION}

To evaluate the biological role of 17,18 -EpETE in the regulation of other types of allergic inflammatory disease, we examined the effect of 17,18-EpETE on the regulation of contact hypersensitivity (CHS) in the hapten-induced CHS model. We found that 17,18-EpETE showed both prophylactic and therapeutic anti-inflammatory effects on CHS in mice and cynomolgus macaques (44). 17,18-EpETE did not affect $\mathrm{T}$ cell or dendritic cell functions, including inducible skinassociated lymphoid tissue formation, but it did selectively inhibit neutrophil infiltration into the skin (44). Indeed, 17,18EpETE reduced neutrophil mobility by inhibiting Rac-activation and pseudopod formation in a GPR40-dependent fashion (44). Consistent with this selective influence on neutrophils, GPR40 was highly expressed by neutrophils, but not $\mathrm{T}$ cells or other leukocytes in the skin. It is worth noting that mast cells do not express GPR40; so, given that mast cell degranulation was inhibited by 17,18-EpETE treatment in the food allergy model $(7,44)$, this finding suggests that 17,18 -EpETE inhibits mast cell degranulation indirectly (Figure 2). Of note, the activation of GPR40 in intestinal epithelial cells has been reported to improve intestinal barrier function by enhancing occludin expression (45). Therefore, it is likely that the improvement in intestinal barrier function induced by 17,18-EpETE via GPR40 in epithelial cells led to decreased allergen penetration, which, in turn, resulted in decreased mast cell degranulation and inhibited food allergy development.

\section{STRUCTURE-ACTIVITY RELATIONSHIPS AMONG THE GPR40-DEPENDENT ANTI-ALLERGIC AND ANTI-INFLAMMATION EFFECTS OF 17,18-EPETE}

17,18-EpETE is further metabolized by soluble epoxide hydrolase to 17,18-dihydroxy-eicosatetraenoic acid (17,18-diHETE). However, 17,18-diHETE has little effect on the development of food allergy, and 14,15-epoxyeicosatetraenoic acid $(14,15-$ EpETE), which has an epoxy structure at the $\omega 6$ position, also lacks the ability to inhibit food allergy (7). In addition,
17,18-diHETE has little effect on the development of CHS (44). Although 17,18-EpETE activates GPR40, 17,18-diHETE does not activate GPR40, which is consistent with its lack of anti-allergic and anti-inflammatory properties $(7,44)$. These findings therefore suggest that the 17,18-epoxy ring structure at the $\omega 3$ position in EPA is important for GPR40-mediated anti-allergic and anti-inflammatory activity.

17,18-EpETE is synthesized from EPA through the enzymatic activity of CYP and has two isomers, 17(S),18(R)-EpETE and $17(R), 18(S)$-EpETE. Among the CYP subfamilies in mice, five CYP isoforms (Cyp1a2, 2c50, 4a12a, 4a12b, and 4f18) are known to convert EPA into 17,18-EpETE (46). Cyp1a2 displays high stereoselectivity for producing $17(R), 18(S)$ EpETE, whereas Cyp4f18 displays stereoselectivity for producing 17(S),18(R)-EpETE (46). In contrast, Cyp2c50, Cyp4a12a, and Cyp4a12b display less stereoselectivity and produce a mixture of 17(S),18(R)-EpETE and 17(R),18(S)-EpETE (46). 17(R),18(S)EpETE, but not $17(S), 18(R)$-EpETE, is a potent vasodilator (47). Indeed, 17(R),18(S)-EpETE activates calcium-activated potassium channels, which lead to relaxation of rat cerebral artery vascular smooth muscle cells (47). Whether stereoselectivity of 17,18-EpETE contributes to the anti-allergy and antiinflammatory effects of 17,18-EpETE have not been evaluated in food allergy and CHS, because we used racemic compounds in our studies $(7,44)$. The CYP isoform and polymorphisms determine the metabolic properties of CYP and stereoselectivity. Therefore, the anti-allergic and anti-inflammatory health benefits derived from $\omega 3$-PUFA intake may be influenced by the expression levels of the various types of CYP in the body.

CYP is also found in microorganisms. For example, it has been reported that bacterial CYP (e.g., BM-3 derived from Bacillus megateirum) metabolizes PUFAs and produces hydroxy and epoxy fatty acids (48). Bacillus, Streptomyces, Pseudomonas, and Mycobacterium also have CYP (49-53). These findings suggest that many types of microorganisms are involved in lipid metabolism. In addition, other metabolic enzymes, such as COX and LOX, are thought to be expressed by some bacteria, including Pseudomonas aeruginosa, Shewanella woodyi, Mytococcus fulrus, and Burkholderia thailandensis $(54,55)$. Some microorganisms described above are present in environment, suggesting that in addition to mammalian expression of metabolic enzymes, various microorganisms may be a determinant of the efficacy of $\omega 3$-PUFA in the context of the regulation of inflammation.

\section{BACTERIAL-CONJUGATED LINOLEIC ACID HAS A ROLE IN ANTI-INFLAMMATION}

Intestinal bacteria have been shown to express unique unsaturated fatty acid-metabolic enzymes and to produce bioactive lipid mediators that are not generated by mammalian cells (Figure 3). Ruminal bacteria including Butyrivibrio, Lactobacillus, and Megasphaera can produce conjugated linoleic acid (CLA), which is an isomer of linoleic acid that has conjugated double bounds (56-58). It is known that CLA 


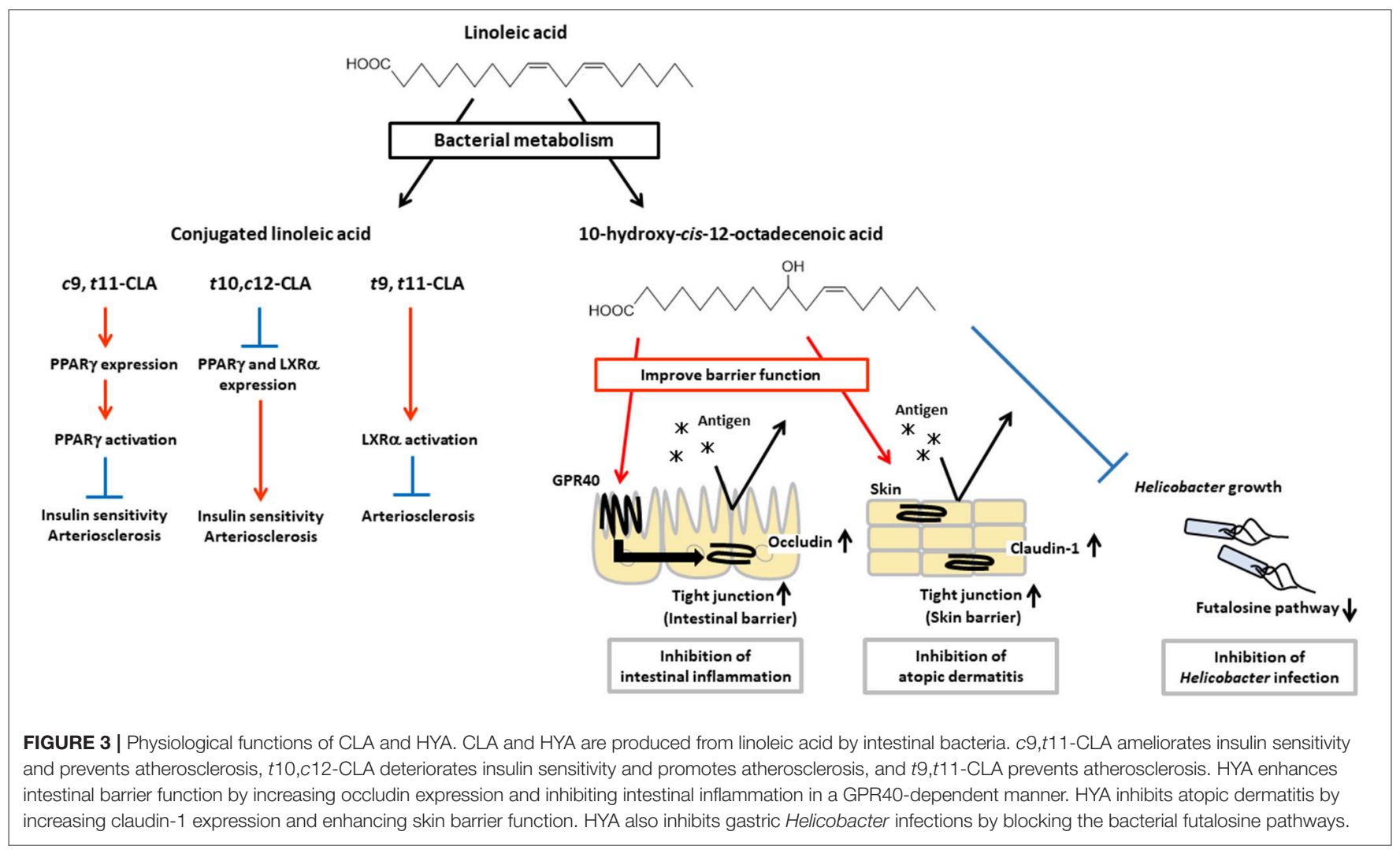

has some isomers such as cis-9-trans-11-octadecenoic acid (c9,t11-CLA), trans-10-cis-12-octadecenoic acid ( $t 10, c 12-$ CLA) and trans-9-trans-11-octadecenoic acid (t9,t11-CLA). These isomers have different activities for insulin sensitivity and atherosclerosis.

For example, $c 9, t 11-$ CLA shows beneficial effects on insulin sensitivity by enhancing glucose uptake and adipokine production such as leptin and adiponectin, and on atherosclerosis by suppressing macrophage infiltration and activation, and reducing plaque development through an increase in expression of PPAR $\gamma$, while t10,c12-CLA shows adverse effects through a decrease in expression of PPAR $\gamma$ (59-63). In addition, $t 10, c 12-C L A$ reduces expression of liver $\mathrm{X}$ receptor $\alpha(\mathrm{LXR} \alpha)$ which induces expression of ATPbinding cassette $(\mathrm{ABC})$ transporter $\mathrm{A} 1, \mathrm{ABCG} 1$, and sterol regulatory element binding protein $1 \mathrm{c}$ which involved in reverse cholesterol transport $(64,65)$. Therefore, $t 10, c 12$-CLA shows pro-atherosclerosis effects (66-68). On the other hand, $t 9, t 11$ CLA is effective for the treatment of atherosclerosis by activation of $\operatorname{LXR} \alpha$ (69). These results indicate that each isomers exert different bioactivities through distinct transcriptional regulation and activation of PPAR $\gamma$ and LXR $\alpha$ for the control of insulin sensitivity and atherosclerosis.

Compared with chemical production, microbial fermentation offers better ways to produce isomer-specific CLAs. The CLA isomers are produced at different ratios, depending on the type of bacteria. Lactobacillus strains (L. acidophilus, L. plantarum, L. casei, L. reuteri, L. rhamnosus, and $L$. pentosus), Bifidobacterium strains (B. dentium, B. breve, and B. lactis), and Propionibacterium freudenreichii can convert linoleic acid to $c 9, t 11-C L A$ and $t 10, c 12-C L A$, and these bacteria produce higher levels of $c 9, t 11$-CLA than of $t 10, c 12$ CLA (15, 57, 70-72). Some Lactobacillus and Bifidobacterium strains also produce $t 9, t 11-$ CLA with $c 9, t 11-$ CLA and/or t10,c12-CLA (57). L. paracasei and B. bifidum produce c9,t11-CLA stereoselectively, whereas Megasphaera eldsenii produces $t 10, c 12-C L A$ stereoselectively $(71,73)$. Given that these CLAs have different biological activities which depend on their 3D-structure, it is important to select appropriate bacteria as a probiotics or producer for obtaining required beneficial effects.

\section{BACTERIAL PRODUCTION OF UNIQUE HYDROXY AND OXO FATTY ACIDS AND THEIR MULTIPLE BIOLOGICAL ACTIVITIES}

L. plantarum, an intestinal bacteria, produces hydroxy fatty acids (i.e., 10-hydroxy-cis-12-octadecenoic acid [HYA], 10-hydroxytrans-11-octadecenoic acid [HYC], 10-hydroxy-octadecanoic acid $[\mathrm{HYB}]$ ) and oxo fatty acids (10-oxo-cis-12-octadecenoic acid [KetoA], 10-oxo-trans-11-octadecenoic acid [KetoC], 10oxo-octadecanoic acid [KetoB]) as intermediate products of CLA production (16). Recently, these metabolic intermediates have been shown to contribute to the regulation of host health and diseases. HYA is the first metabolite produced 
from linoleic acid by L. plantarum, and it enhances intestinal barrier function and suppresses the development of DSSinduced colitis in mice in a GPR40-dependent manner (45). Furthermore, HYA prevents Helicobacter infections by blocking their futalosine pathways, which is an alternative menaquinone biosynthetic pathway and an essential metabolic pathway for the growth of Helicobacter. Moreover, HYA treatment suppresses the formation of lymphoid follicles in the gastric mucus layer after $H$. suis infection, and therefore HYA treatment protects mice against the formation of gastric mucosaassociated lymphoid tissue lymphoma induced by infection with Helicobacter (74). HYA also ameliorates the pathological scores of atopic dermatitis in NC/Nga mice by decreasing plasma IgE levels and reducing mast cell infiltration into the skin $(75,76)$. KetoA enhances adiponectin production and glucose uptake in a proliferator-activated receptor $\gamma$ $(\operatorname{PPAR} \gamma)$-dependent manner, and is effective for the prevention and amelioration of metabolic abnormalities associated with obesity (77).

The production of these hydroxy and oxo fatty acids depends on the unique bacterial enzymes CLA-HY (unsaturated fatty acid hydratase), CLA-DH (hydroxy fatty acid dehydrogenase), CLADC (isomerase), and CLA-ER (enone reductase) in L. plantarum AKU1009a $(16,78)$. The hydroxy activity is found not only in Lactobacillus but also in a broad spectrum of bacteria. Oleate hydratase belongs to the FAD-dependent myosin crossreactive antigen (MCRA) protein family, which is found in grampositive and -negative bacteria; it catalyzes the conversion of linoleic acid to HYA. For example, Lactobacillus, Bifidobacterium, Streptococcus, and Stenotrophomonas bacteria are reported to have MCRA, and indeed they have the ability to produce HYA (79-82).

Together, these findings indicate that intestinal bacteria metabolize dietary lipids and produce lipid metabolites that can regulate host immune systems. Therefore, to obtain beneficial lipid metabolites and regulate intestinal inflammation, we need to consider not only host enzymes but also enzymes produced by intestinal bacteria. In addition, we must consider how dietary lipid intake causes changes in the intestinal microbiota.

\section{REFERENCES}

1. Martins D, Custódio L, Barreira L, Pereira H, Ben-Hamadou R, Varela J, et al. Alternative sources of n-3 long-chain polyunsaturated fatty acids in marine microalgae. Mar Drugs. (2013) 11:2259-81. doi: 10.3390/md11072259

2. Vrinten P, Hu Z, Munchinsky MA, Rowland G, Qiu X. Two FAD3 desaturase genes control the level of linolenic acid in flax seed. Plant Physiol. (2005) 139:79-87. doi: 10.1104/pp.105.064451

3. Lee JM, Lee H, Kang S, Park WJ. Fatty acid desaturases, polyunsaturated fatty acid regulation, and biotechnological advances. Nutrients. (2016) 8:23. doi: $10.3390 /$ nu8010023

4. Dyerberg J, Bang HO, Stoffersen E, Moncada S, Vane JR. Eicosapentaenoic acid and prevention of thrombosis and atherosclerosis? Lancet. (1978) 312:117-9. doi: 10.1016/S0140-6736(78)91505-2

5. Mozaffarian D, Rimm EB. Fish intake, contaminants, and human health: evaluating the risks and the benefits. JAMA. (2006) 296:1885-99. doi: 10.1001/jama.296.15.1885

\section{CONCLUSION}

Recent technological developments in lipidomics research initiated a new era of lipid biology by helping researchers to identify novel lipid metabolites from $\omega 3$ - and $\omega 6$-PUFAs, which actively regulate the host immune system and play important roles in the control of health and diseases. Given that the production of lipid metabolites is influenced by complex factors, including diet, intestinal bacteria, and enzyme expression, combined studies on nutrition, metabolomics, and the metagenomics of the microbiota, as well as informatics, may provide powerful insights to further our understanding of the lipid network in the host immune system.

\section{AUTHOR CONTRIBUTIONS}

All authors listed have made a substantial, direct and intellectual contribution to the work, and approved it for publication.

\section{ACKNOWLEDGMENTS}

We thank laboratory members for helpful discussion. The results described in the review were obtained, at least in part, from research supported by grants from the Ministry of Education, Culture, Sports, Science, and Technology of Japan (MEXT) and the Japan Society for the Promotion of Science (JSPS; KAKENHI [JP15K19142 to TN; JP15H05790, JP18H02150, JP18H02674, JP17K09604, JP26670241, and JP26293111 to JK]); the Japan Agency for Medical Research and Development (AMED; [JP17ek0410032s0102, JP17ek0210078h0002, JP17ak0101068h0001, JP17gm1010006s 0101, JP18ck0106243h0003, and 19ek0410062h0001 to JK]); the Ministry of Health and Welfare of Japan (to JK); the Science and Technology Research Promotion Program for Agriculture, Forestry, Fisheries, and Food Industry (to JK); grants-in-aid for Scientific Research on Innovative Areas from MEXT (JP23116506, JP16H01373, and JP25116706 to JK); Cross-ministerial Strategic Innovation Promotion Program (SIP); the Ono Medical Research Foundation; and the Canon Foundation (to JK).

6. Miyata J, Arita M. Role of omega-3 fatty acids and their metabolites in asthma and allergic diseases. Allergol Int. (2015) 64:27-34. doi: 10.1016/j.alit.2014.08.003

7. Kunisawa J, Arita M, Hayasaka T, Harada T, Iwamoto R, Nagasawa R, et al. Dietary omega3 fatty acid exerts anti-allergic effect through the conversion to 17,18 -epoxyeicosatetraenoic acid in the gut. Sci Rep. (2015) 5:9750. doi: 10.1038/srep09750

8. Yoshida S, Yasutomo K, Watanabe T. Treatment with DHA/EPA ameliorates atopic dermatitis-like skin disease by blocking $\mathrm{LTB}_{4}$ production. J Med Invest. (2016) 63:187-91. doi: 10.2152/jmi.63.187

9. Del Gobbo LC, Imamura F, Aslibekyan S, Marklund M, Virtanen JK, Wennberg $\mathrm{M}$, et al. $\omega-3$ polyunsaturated fatty acid biomarkers and coronary heart disease: pooling project of 19 cohort studies. JAMA Intern Med. (2016) 176:1155-66. doi: 10.1001/jamainternmed.2016.2925

10. Wang $M$, Zhang $X$, Ma LJ, Feng RB, Yan C, Su H, et al. Omega3 polyunsaturated fatty acids ameliorate ethanol-induced adipose hyperlipolysis: a mechanism for hepatoprotective effect against alcoholic 
liver disease. Biochim Biophys Acta Mol Basis Dis. (2017) 1863:3190-201. doi: 10.1016/j.bbadis.2017.08.026

11. Alshatwi AA, Subash-Babu P. Effects of increasing ratios of dietary omega6/omega-3 fatty acids on human monocyte immunomodulation linked with atherosclerosis. J Funct Foods. (2018) 41:258-67. doi: 10.1016/j.jff.2017.12.020

12. Calder PC. Very long-chain $\mathrm{n}-3$ fatty acids and human health: fact, fiction and the future. Proc Nutr Soc. (2018) 77:52-72. doi: 10.1017/S0029665117 003950

13. Hirakata T, Lee HC, Ohba M, Saeki K, Okuno T, Murakami A, et al. Dietary omega-3 fatty acids alter the lipid mediator profile and alleviate allergic conjunctivitis without modulating $\mathrm{T}_{\mathrm{h}} 2$ immune responses. FASEB J. (2018) 33:3392-403. doi: 10.1096/fj.201801805R

14. Serhan CN. Treating inflammation and infection in the 21st century: new hints from decoding resolution mediators and mechanisms. FASEB J. (2017) 31:1273-88. doi: 10.1096/fj.201601222R

15. Nieuwenhove CPV, Terán V, González SN. Conjugated Linoleic and Linolenic Acid Production by Bacteria: Development of Functional Foods. In: E Rigobelo, editor. Probiotics. London: IntechOpen (2012). doi: 10.5772/50321

16. Kishino S, Takeuchi M, Park SB, Hirata A, Kitamura N, Kunisawa J, et al. Polyunsaturated fatty acid saturation by gut lactic acid bacteria affecting host lipid composition. Proc Natl Acad Sci USA. (2013) 110:17808-13. doi: 10.1073 /pnas. 1312937110

17. Zárate R, el Jaber-Vazdekis N, Tejera N, Pérez JA, Rodríguez C. Significance of long chain polyunsaturated fatty acids in human health. Clin Transl Med. (2017) 6:25. doi: 10.1186/s40169-017-0153-6

18. Hicks A, Monkarsh SP, Hoffman AF, Goodnow Jr R. Leukotriene $B_{4}$ receptor antagonists as therapeutics for inflammatory disease: preclinical and clinical developments. Expert Opin Investig Drugs. (2007) 16:1909-20. doi: 10.1517/13543784.16.12.1909

19. Yokomizo T. Two distinct leukotriene $\mathrm{B}_{4}$ receptors, BLT1 and BLT2. $J$ Biochem. (2014) 157:65-71. doi: 10.1093/jb/mvu078

20. Saeki K, Yokomizo T. Identification, signaling, and functions of $\mathrm{LTB}_{4}$ receptors. Semin Immunol. (2017) 33:30-6 doi: 10.1016/j.smim.2017.07.010

21. Ishii Y, Saeki K, Liu M, Sasaki F, Koga T, Kitajima K, et al. Leukotriene $B_{4}$ receptor type 2 (BLT2) enhances skin barrier function by regulating tight junction proteins. FASEB J. (2015) 30:933-47. doi: 10.1096/fj.15-279653

22. Sturm EM, Radnai B, Jandl K, Stančić A, Parzmair GP, Högenauer C, et al. Opposing roles of prostaglandin $\mathrm{D}_{2}$ receptors in ulcerative colitis. J Immunol. (2014) 193:827-39. doi: 10.4049/jimmunol.1303484

23. Radnai B, Sturm EM, Stančić A, Jandl K, Labocha S, Ferreirós $N$, et al. Eosinophils contribute to intestinal inflammation via chemoattractant receptor-homologous molecule expressed on Th2 cells, CRTH2, in experimental Crohn's disease. J Crohns Colitis. (2016) 10:1087-95. doi: 10.1093/ecco-jcc/jjw061

24. Ajuebor MN, Singh A, Wallace JL. Cyclooxygenase-2-derived prostaglandin $\mathrm{D}_{2}$ is an early anti-inflammatory signal in experimental colitis. Am J Physiol Gastrointest Liver Physiol. (2000) 279:G238-44. doi: 10.1152/ajpgi.2000.279.1.G238

25. Zamuner SR, Bak AW, Devchand PR, Wallace JL. Predisposition to colorectal cancer in rats with resolved colitis: role of cyclooxygenase2-derived prostaglandin $\mathrm{d}_{2}$. Am $J$ Pathol. (2005) 167:1293-300. doi: 10.1016/S0002-9440(10)61216-3

26. Kupczyk M, Kuna P. Targeting the $\mathrm{PGD}_{2} / \mathrm{CRTH} 2 / \mathrm{DP} 1$ signaling pathway in asthma and allergic disease: current status and future perspectives. Drugs. (2017) 77:1281-94. doi: 10.1007/s40265-017-0777-2

27. Ma X, Aoki T, Tsuruyama T, Narumiya S. Definition of prostaglandin E2-EP2 signals in the colon tumor microenvironment that amplify inflammation and tumor growth. Cancer Res. (2015) 75:2822-2832. doi: 10.1158/0008-5472.CAN-15-0125

28. Sarashina H, Tsubosaka Y, Omori K, Aritake K, Nakagawa T, Hori M, et al. Opposing immunomodulatory roles of prostaglandin $\mathrm{D}_{2}$ during the progression of skin inflammation. J Immunol. (2014) 192:459-65. doi: 10.4049/jimmunol.1302080

29. Kostenis E, Ulven T, Emerging roles of DP and CRTH2 in allergic inflammation. Trends Mol Med. (2006) 12:148-58. doi: 10.1016/j.molmed.2006.02.005

30. Bargut TCL, Ferreira TPT, Daleprane JB, Martins MA, Silva PMR, Aguila MB. Fish oil has beneficial effects on allergen-induced airway inflammation and hyperreactivity in mice. PLoS ONE. (2013) 8:e75059. doi: 10.1371/journal.pone.0075059
31. Van Den Elsen LW, Bol-Schoenmakers M, van Esch BC, Hofman GA, van de Heijning BJ, Pieters RH, et al. DHA-rich tuna oil effectively suppresses allergic symptoms in mice allergic to whey or peanut. J. Nutr. (2014) 144:1970-6. doi: 10.3945/jn.114.198515

32. Shoda H, Yanai R, Yoshimura T, Nagai T, Kimura K, Sobrin L, et al. Dietary omega-3 fatty acids suppress experimental autoimmune uveitis in association with inhibition of Th1 and Th17 cell function. PLOS ONE. (2015) 10:e0138241. doi: 10.1371/journal.pone.0138241

33. Bisgaard H, Stokholm J, Chawes BL, Vissing NH, Bjarnadóttir E, Schoos AMM, et al. Fish oil-derived fatty acids in pregnancy and wheeze and asthma in offspring. N Engl J Med. (2016) 375:2530-9. doi: 10.1056/NEJMoa1503734

34. Bi X, Li F, Liu S, Jin Y, Zhang X, Yang T. et al. $\omega-3$ polyunsaturated fatty acids ameliorate type 1 diabetes and autoimmunity. J. Clin. Invest. (2017) 127:1757-71. doi: 10.1172/JCI87388

35. Levy BD. Resolvin D1 and resolvin E1 promote the resolution of allergic airway inflammation via shared and distinct molecular counter-regulatory pathways. Front Immunol. (2012) 3:390. doi: 10.3389/fimmu.2012.00390

36. Rogerio AP, Haworth O, Croze R, Oh SF, Uddin M, Carlo T, et al. Resolvin D1 and aspirin-triggered resolvin D1 promote resolution of allergic airways responses. J Immunol. (2012) 189:1983-91. doi: 10.4049/jimmunol.1101665

37. Serhan CN, Chiang N, Dalli J, Levy BD. Lipid mediators in the resolution of inflammation. Cold Spring Harb Perspect Biol. (2015) 7:a016311. doi: 10.1101/cshperspect.a016311

38. Arita M, Ohira T, Sun YP, Elangovan S, Chiang N, Serhan CN. Resolvin E1 selectively interacts with leukotriene $\mathrm{B}_{4}$ receptor BLT1 and ChemR23 to regulate inflammation. J Immunol. (2007) 178:3912-7. doi: 10.4049/jimmunol.178.6.3912

39. Krishnamoorthy S, Recchiuti A, Chiang N, Yacoubian S, Lee CH, Yang R, et al. Resolvin D1 binds human phagocytes with evidence for proresolving receptors. Proc Natl Acad Sci USA. (2010) 107:1660-5. doi: 10.1073/pnas.0907342107

40. Ishida T, Yoshida M, Arita M, Nishitani Y, Nishiumi S, Masuda A, et al. Resolvin E1, an endogenous lipid mediator derived from eicosapentaenoic acid, prevents dextran sulfate sodium-induced colitis. Inflamm Bowel Dis. (2010) 16:87-95. doi: 10.1002/ibd.21029

41. Schwab JM, Chiang N, Arita M, Serhan CN. Resolvin E1 and protectin D1 activate inflammation-resolution programmes. Nature. (2007) 447:869-74. doi: 10.1038/nature05877

42. Schmid M, Gemperle C, Rimann N, Hersberger M. Resolvin D1 polarizes primary human macrophages toward a proresolution phenotype through GPR32. J Immunol. (2016) 196:3429-37. doi: 10.4049/jimmunol.1501701

43. Marcon R, Bento AF, Dutra RC, Bicca MA, Leite DF, Calixto JB. Maresin 1, a proresolving lipid mediator derived from omega-3 polyunsaturated fatty acids, exerts protective actions in murine models of colitis. J Immunol. (2013) 191:4288-98. doi: 10.4049/jimmunol.1202743

44. Nagatake T, Shiogama Y, Inoue A, Kikuta J, Honda T, Tiwari P, et al. The 17,18-epoxyeicosatetraenoic acid-G protein-coupled receptor 40 axis ameliorates contact hypersensitivity by inhibiting neutrophil mobility in mice and cynomolgus macaques. J Allergy Clin Immunol. (2018) 142:470-84. doi: $10.1016 /$ j.jaci.2017.09.053

45. Miyamoto J, Mizukure T, Park SB, Kishino S, Kimura I, Hirano K, et al. A gut microbial metabolite of linoleic acid, 10-hydroxy-cis-12octadecenoic acid, ameliorates intestinal epithelial barrier impairment partially via GPR40-MEK-ERK pathway. J Biol Chem. (2015) 290:2902-18. doi: 10.1074/jbc.M114.610733

46. Isobe $\mathrm{Y}$, Itagaki $\mathrm{M}$, Ito $\mathrm{Y}$, Naoe $\mathrm{S}$, Kojima $\mathrm{K}$, Ikeguchi $\mathrm{M}$, et al. Comprehensive analysis of the mouse cytochrome P450 family responsible for omega-3 epoxidation of eicosapentaenoic acid. Sci Rep. (2018) 8:7954. doi: 10.1038/s41598-018-26325-4

47. Lauterbach B, Barbosa-Sicard E, Wang MH, Honeck H, Kärgel E, Theuer J, et al. Cytochrome P450-dependent eicosapentaenoic acid metabolites are novel BK channel activators. Hypertension. (2002) 39:609-13. doi: 10.1161/hy0202.103293

48. Capdevila JH, Wei S, Helvig C, Falck JR, Belosludtsev Y, Truan G, et al. The highly stereoselective oxidation of polyunsaturated fatty acids by cytochrome P450BM-3. J Biol Chem. (1996) 271:22663-71. doi: 10.1074/jbc.271.37.22663

49. Lamb DC, Lei L, Zhao B, Yuan H, Jackson CJ, Warrilow AG, et al. Streptomyces coelicolor A3 (2) CYP102 protein, a novel fatty acid hydroxylase encoded as a heme domain without an N-terminal redox partner. Appl Environ Microbiol. (2010) 76:1975-80. doi: 10.1128/AEM.03000-09 
50. Martínez E, Hamberg M, Busquets M, Díaz P, Manresa A, Oliw EH. Biochemical characterization of the oxygenation of unsaturated fatty acids by the dioxygenase and hydroperoxide isomerase of Pseudomonas aeruginosa 42A2. J Biol Chem. (2010) 285:9339-45. doi: 10.1074/jbc.M109.078147

51. Van Bogaert IN, Groeneboer S, Saerens K, Soetaert W. The role of cytochrome P450 monooxygenases in microbial fatty acid metabolism. FASEB J. (2011) 278:206-21. doi: 10.1111/j.1742-4658.2010.07949.x

52. Kelly SL, Kelly DE. Microbial cytochromes P450: biodiversity and biotechnology. Where do cytochromes P450 come from, what do they do and what can they do for us? Philos Trans R Soc Lond B. Biol Sci. (2013) 368:20120476. doi: 10.1098/rstb.2012.0476

53. Parvez M, Qhanya LB, Mthakathi NT, Kgosiemang IKR, Bamal HD, Pagadala NS, et al. Molecular evolutionary dynamics of cytochrome P450 monooxygenases across kingdoms: special focus on mycobacterial P450s. Sci Rep. (2016) 6:33099. doi: 10.1038/srep33099

54. Hansen J, Garreta A, Benincasa M, Fusté MC, Busquets M, Manresa A. Bacterial lipoxygenases, a new subfamily of enzymes? a phylogenetic approach. Appl Microbiol Biotechnol. (2013) 97:4737-47. doi: 10.1007/s00253-013-4887-9

55. An JU, Kim BJ, Hong SH, Oh DK. Characterization of an omega-6 linoleate lipoxygenase from Burkholderia thailandensis and its application in the production of 13-hydroxyoctadecadienoic acid. Appl Microbiol Biotechnol. (2015) 99:5487-97. doi: 10.1007/s00253-014-6353-8

56. Kim YJ, Liu RH. Increase of conjugated linoleic acid content in milk by fermentation with lactic acid bacteria. J Food Sci Technol. (2002) 67:1731-7. doi: 10.1111/j.1365-2621.2002.tb08714.x

57. Ogawa J, Kishino S, Ando A, Sugimoto S, Mihara K, Shimizu S. Production of conjugated fatty acids by lactic acid bacteria. J Biosci Bioeng. (2005) 100:355-64. doi: 10.1263/jbb.100.355

58. Puniya AK, Chaitanya S, Tyagi AK, De S, Singh K. Conjugated linoleic acid producing potential of lactobacilli isolated from the rumen of cattle. $J$ Ind Microbiol Biotechnol. (2008) 35:1223-8. doi: 10.1007/s10295-008-0429-3

59. Brown JM, Mclntosh MK. Conjugated linoleic acid in humans: regulation of adiposity and insulin sensitivity. J Nutr. (2003) 133:3041-6. doi: 10.1093/jn/133.10.3041

60. Wargent E, Sennitt MV, Stocker C, Mayes AE, Brown L, O’Dowd J, et al. Prolonged treatment of genetically obese mice with conjugated linoleic acid improves glucose tolerance and lowers plasma insulin concentration: possible involvement of PPAR activation. Lipids Health Dis. (2005) 4:3. doi: 10.1186/1476-511X-4-3

61. Leonardini A, Laviola L, Perrini S, Natalicchio A, Giorgino F. Cross-talk between PPAR and insulin signaling and modulation of insulin sensitivity. PPAR Res. (2009) 2009:818945. doi: 10.1155/2009/818945

62. Rubin D, Herrmann J, Much D, Pfeuffer M, Laue C, Winkler P, et al. Influence of different CLA isomers on insulin resistance and adipocytokines in prediabetic, middle-aged men with PPAR $\gamma 2$ Pro12Ala polymorphism. Genes Nutr. (2012) 7:499-509. doi: 10.1007/s12263-012-0289-3

63. Brown JM, Boysen MS, Jensen SS, Morrison RF, Storkson J, Lea-Currie $\mathrm{R}$, et al. Isomer-specific regulation of metabolism and PPAR $\gamma$ signaling by CLA in human preadipocytes. J Lipid Res. (2003) 44:1287-300. doi: 10.1194/jlr.M300001-JLR200

64. Granlund L, Juvet LK, Pedersen JI, Nebb HI. Trans10, cis12-conjugated linoleic acid prevents triacylglycerol accumulation in adipocytes by acting as a PPAR $\gamma$ modulator. J Lipid Res. (2003) 44:1441-52. doi: 10.1194/jlr.M300120-JLR200

65. Ecker J, Langmann T, Moehle C, Schmitz G. Isomer specific effects of conjugated linoleic acid on macrophage ABCG1 transcription by a SREBP1c dependent mechanism. Biochem Biophys Res Commun. (2007) 352:805-11. doi: 10.1016/j.bbrc.2006.11.087

66. Arbonés-Mainar JM, Navarro MA, Guzmán MA, Arnal C, Surra JC, Acín S, et al. Selective effect of conjugated linoleic acid isomers on atherosclerotic lesion development in apolipoprotein E knockout mice. Atherosclerosis. (2006) 189:318-27. doi: 10.1016/j.atherosclerosis.2006.01.015

67. Toomey S, Harhen B, Roche HM, Fitzgerald D, Belton O. Profound resolution of early atherosclerosis with conjugated linoleic acid. Atherosclerosis. (2006) 187:40-9. doi: 10.1016/j.atherosclerosis.2005.08.024

68. de Gaetano M, Alghamdi K, Marcone S, Belton O. Conjugated linoleic acid induces an atheroprotective macrophage $\mathrm{M} \Phi 2$ phenotype and limits foam cell formation. I Inflamm. (2015) 12:15. doi: 10.1186/s12950-0150060-9

69. Ecker J, Liebisch G, Patsch W, Schmitz G. The conjugated linoleic acid isomer trans-9, trans-11 is a dietary occurring agonist of liver $\mathrm{X}$ receptor $\alpha$. Biochem Biophys Res Commun. (2009) 388:660-6. doi: 10.1016/j.bbrc.2009. 08.048

70. Xu S, Boylston TD, Glatz BA. Effect of lipid source on probiotic bacteria and conjugated linoleic acid formation in milk model systems. J Am Oil Chem Soc. (2004) 81:589-95. doi: 10.1007/s11746-006-0946-Z

71. Lee HY, Park JH, Seok SH, Baek MW, Kim DJ, Lee KE, et al. Human originated bacteria, Lactobacillus rhamnosus PL60, produce conjugated linoleic acid and show anti-obesity effects in diet-induced obese mice. Biochim Biophys Acta Mol Cell Biol Lipids. (2006) 1761:736-44. doi: 10.1016/j.bbalip.2006.05.007

72. Coakley M, Ross RP, Nordgren M, Fitzgerald G, Devery R, Stanton C. Conjugated linoleic acid biosynthesis by humanderived Bifidobacterium species. I Appl Microbiol. (2003) 94:138-45. doi: 10.1046/j.1365-2672.2003.01814.x

73. Kim TW, Choi NJ, Hwangbo J, Hsu JT, Lee SS, Song MK, et al. Production of trans-10, cis-12 conjugated linoleic acid by Megasphaera elsdenii YJ4: physiological roles in the rumen. Asian-australas J Anim Sci. (2005) 18:1425-9. doi: 10.5713/ajas.2005.1425

74. Matsui H, Takahashi T, Murayama SY, Kawaguchi M, Matsuo K, Nakamura M. Protective efficacy of a hydroxy fatty acid against gastric Helicobacter infections. Helicobacter. (2017) 22:e12430. doi: 10.1111/hel.12430

75. Kaikiri H, Miyamoto J, Kawakami T, Park SB, Kitamura N, Kishino S, et al. Supplemental feeding of a gut microbial metabolite of linoleic acid, 10hydroxy-cis-12-octadecenoic acid, alleviates spontaneous atopic dermatitis and modulates intestinal microbiota in NC/nga mice. Int J Food Sci Nutr. (2017) 68:941-51. doi: 10.1080/09637486.2017.1318116

76. Park HJ, Lee SW, Hong S. Regulation of allergic immune responses by microbial metabolites. Immune Netw. (2018) 18:e15. doi: 10.4110/in.2018.18.e15

77. Goto T, Kim YI, Furuzono T, Takahashi N, Yamakuni K, Yang HE, et al. 10-oxo-12 (Z)-octadecenoic acid, a linoleic acid metabolite produced by gut lactic acid bacteria, potently activates PPAR $\gamma$ and stimulates adipogenesis. Biochem Biophys Res Commun. (2015) 459:597-603. doi: 10.1016/j.bbrc.2015. 02.154

78. Takeuchi M, Kishino S, Park SB, Kitamura N, Watanabe H, Saika A, et al. Production of dicarboxylic acids from novel unsaturated fatty acids by laccasecatalyzed oxidative cleavage. Biosci Biotechnol Biochem. (2016) 80:2132-7. doi: 10.1080/09168451.2016.1200457

79. Hirata A, Kishino S, Park SB, Takeuchi M, Kitamura N, Ogawa J. A novel unsaturated fatty acid hydratase toward C16 to C22 fatty acids from Lactobacillus acidophilus. J Lipid Res. (2015) 56:1340-50. doi: 10.1194/jlr.M059444

80. Chen YY, Liang NY, Curtis JM, Gänzle MG. Characterization of linoleate 10hydratase of Lactobacillus plantarum and novel antifungal metabolites. Front Microbiol. (2016) 7:1561. doi: 10.3389/fmicb.2016.01561

81. Kang WR, Seo MJ, Shin KC, Park JB, Oh DK. Comparison of biochemical properties of the original and newly identified oleate hydratases from Stenotrophomonas maltophilia. J Appl Environ Microbiol. (2017) 83:e03351-16. doi: 10.1128/AEM.03351-16

82. Serra S, Simeis DD. Use of Lactobacillus rhamnosus (ATCC 53103) as whole-cell biocatalyst for the regio-and stereoselective hydration of oleic, linoleic, and linolenic acid. Catalysts. (2018) 8:109. doi: 10.3390/catal80 30109

Conflict of Interest Statement: The authors declare that the research was conducted in the absence of any commercial or financial relationships that could be construed as a potential conflict of interest.

Copyright (c) 2019 Saika, Nagatake and Kunisawa. This is an open-access article distributed under the terms of the Creative Commons Attribution License (CC BY). The use, distribution or reproduction in other forums is permitted, provided the original author(s) and the copyright owner(s) are credited and that the original publication in this journal is cited, in accordance with accepted academic practice. No use, distribution or reproduction is permitted which does not comply with these terms. 